\title{
An Update on the Various Theories of the Origin of Uterine Adenomyosis -A Systematic Review
}

\author{
Dr. Kulvinder Kochar Kaur, MD ${ }^{1}$, Dr. Gautam Allahbadia, MD (Obstt \& Gynae), D.N.B ${ }^{2}$ \\ Dr. Mandeep Singh, MD, DM.(Std) (Neurology) ${ }^{3}$ \\ ${ }^{1}$ Scientific Director, Dr Kulvinder Kaur Centre For Human Reproduction, Punjab, India. \\ ${ }^{2}$ Scientific Director, Ex-Rotunda-A Centre for Human Reproduction, Mumbai, India. \\ ${ }^{3}$ Consultant Neurologist, Swami Satyanand Hospital, Punjab, India. \\ *Corresponding Author: Dr. Kulvinder Kochar Kaur, MD, Scientific Director, Dr Kulvinder Kaur Centre For Human \\ Reproduction, Punjab, India.
}

\begin{abstract}
Adenomyosis is a frequently diagnosed estrogen based gynaecological disorder, whose pathology as well as etiology is not clear. For the origination of Adenomyotic lesions as well as modes implicated in the evolution as well as propagation of the disease, till now two basic theories had been posited like implication of tissue injury as well as repair mechanisms and promote that adenomyosis occurs via invagination of endometrium basalis in the myometrium. Another theory which says that Adenomyotic lesions occurs secondary to metaplasia of displaced embryonic pluripotent mullerian remnants or differentiation of adult stem cells. But as far as Guo's theory, both theories have apparent limitations, i.e., difficulty in falsification, explaining existing data, and making useful predictions. Based on the current understanding of wound healing, a new hypothesis, called endometrial-myometrial interface disruption (EMID), is proposed to account for adenomyosis resulting from iatrogenic trauma to EMI. The EMID hypothesis not only highlights the more salient feature, i.e., hypoxia, at the wounding site, but also incorporates epithelial mesenchymal transition, recruitment of bone-marrowderived stem cells, and enhanced survival and dissemination of endometrial cells dispersed and displaced due to iatrogenic procedures. More importantly, the EMID hypothesis predicts that the risk of adenomyosis can be reduced if certain perioperative interventions are performed. Consequently, from a pathogenic standpoint, adenomyosis is not simply endometriosis of the uterus, and, as such, may call for interventional procedures that are somewhat different from those for endometriosis to achieve the best results.
\end{abstract}

Keywords: TIAR; Adenomyosis; mullerian remnants; adult stem cells; EMID; EMI trauma; collective cell migration.

\section{INTRODUCTION}

Adenomyosis is a usually found to be a benign uterine disease, afflicting $19.5 \%$ of women of reproductive age [1]. Histopathological examination (HPE) shows presence of ectopic endometrial tissue (endometrial glands as well as/or stroma) in the myometrium, surrounded by hyperplastic as well as hypertrophic smooth muscle as well [2]. Various distribution forms are taken up by the ectopic endometrial implants in the myometrium which gives 2 major types of the disease i) focal as well as ii) diffuse. Adenomyosis is labeled focal when a circumscribed nodular form is found but diffuse if a variety of groups of endometrial glands as well as stroma are distributed throughout the myometrium [3]. Conversely in some rare cases Adenomyosis might also be seen as a huge cyst (cystic adenoma)[3,4].

Clinical features are pelvic pain as well as abnormal uterine bleeding (AUB) as well as infertility [5, 6]. but symptoms are not specific and an overlap might exist with different gynaecological diseases like endometriosis as well as a leiomyoma hence interfering with preoperative diagnosis [7,8]. A big advance in diagnosis has come via imaging techniques 
An Update on the Various Theories of the Origin of Uterine Adenomyosis -A Systematic Review

like transvaginal sonography (TVS) as well as magnetic resonance imaging (MRI) which have caused main innovation in forming newer conservative therapies to get formed for treatment of Adenomyosis [9-12]. Nevertheless, the gold standard for the diagnosis of Adenomyosis still remains histological examination following surgery -details by Gordts [8] as well as Bazot [12].

Both endometriosis as well as Adenomyosis are very closely related disease $[4,13]$. The rate of coexistence differs as per endometriotic phenotype that is involved, like shown recently by Chapron et al as well as Leyendecker $[4,5,13]$. They further have a lot of common features, symptomatology, as well as molecular changes [14-16]. Still lot of variations is there in their etiopathogenesis as well as pathogenic mediators[17].

Inspite of prevalence as well as severe symptoms not much is known regarding the etiology as well as pathogenesis of Adenomyosis.

\section{MeTHODS}

We conducted a pubmed review of different theories suggested regarding the etiopathogenesis of Adenomyosis using the MeSH terms Adenomyosis; endometriosis; commonality of etiopathogenesis; uterine stem cells; invasion; estrogen levels; progesterone alterations; metaplasia from 1990 till date in may 2020.

\section{RESULTS}

We found a total of 2742 articles out of which we selected 84 articles for this review.

\section{Posit on Adenomyosis Origin}

Despite etiology as well as pathogenesis not being clear 2 basic theories have been posited in the literature namely i) invagination of the endometrial basalis due to activation of the tissue injury as well as repair (TIAR) mechanism as well as metaplasia of displaced embryonic pluripotent mullerian remnants or differentiation of adult stem cells.

\section{Invagination of the Endometrial Basalis}

Steroid hormones have a main part in the etiology of adenomyosis. Actually supraphysiological estrogen synthesis (hyperestrogenism) secondary to local paracrine action in eutopic as well as ectopic endometrium of patients presenting with adenomyosis might be the initial status, that aid in the development of the disease. This concept gets corroborated by the escalated amounts of E2, usually observed in the menstrual blood of women with adenomyosis as compared to peripheral blood amounts [18].

Gene polymorphisms =>enhanced synthesis (aromatase cytochrome P450-1B1 [CYP1 B1]432 $\mathrm{C} / \mathrm{G}$ as well as cyclooxygenase-2[COX-2] $1195 \mathrm{G} / \mathrm{A}$ ) as well as decreased metabolism (catechol-0-methyl transferase [COMT] 158 G/A) of estrogens are correlated with a > risk of adenomyosis formation $[19,20]$. Due to this hyperestrogenism is pointed to occur due to escalated local aromatization as well as decreased local estrogen metabolism in the eutopic as well as ectopic endometrium of patients presenting with Adenomyosis. Actually Aromatase cytochrome $\mathrm{P} 450$, which is a heme -containing enzyme that catalyzes the reactions involved in steroidogenesis, is not present within the endometrium of disease -free uteri as shown by Kitawasi et al. However it is present in the eutopic endometrium of patients presenting with adenomyosis, that facilitates estrogens biosynthesis with greater bioavailability because of local aromatization of circulating androgens (T) into E2 [21]. Decreased E2 conversion to less potent estrone was also seen in eutopic as well as ectopic endometrium of patients presenting with Adenomyosis, due to decreased expression of 17 $\beta$ hydroxy steroid dehydrogenase type2(17 $\beta$ HSD2) enzyme[22].

Estrogen stimulated endometrium proliferation in healthy endometrium gets counteracted by $\mathrm{P}$ but not in patients of Adenomyosis. In these cases, stromal cells of the endometrial functionalis as well as basalis demonstrate lower immunoreactivity for isoform $B$ of the $\mathrm{P}$ receptor (PR-B)in eutopic endometrium as compared to disease free endometrium, resulting in loss of its action and ultimately a mode of $\mathrm{P}$ resistance [23, 24]. Thus during secretory phase of cycle, estrogen-driven proliferative actions on endometrium are not fully equipped via $\mathrm{P}$, resulting in abnormal endometrium proliferation. Further in Adenomyosis, hyperestrogenism might facilitate escalated oxytocin modulated uterine activity, causing escalated mechanical strains as well as stresses which could injure cells in the junctional zone(JZ)B next to the fundocornual raphe $[13,25,26]$. Changed endometrium proliferation as well as hyperperistalsis -induced tissue microtrauma in the JZ secondary to 
An Update on the Various Theories of the Origin of Uterine Adenomyosis -A Systematic Review

supraphysiologic estrogen synthesis might hence escalate endometrial myometrial invagination (fig1 $\mathrm{A}$ as well as 2A) [see review 27].

Actually, as proof of tissue microtrauma, amounts of antismooth muscle antibody positive as well as collagen 1 positive myofibroblasts are significantly greater in the JZ of women with Adenomyosis as compared to those without [28].

The TIAR mode then gets activated secondary to tissue autotraumatiization $[13,25,26]$. This mode results in a particular physiologic process which facilitates local synthesis of interleukin 1 as well as induces activation of COX-2, leading to synthesis of prostaglandin E2, steroidogenic acute regulatory proteins (STAR) as well as P450 aromatase are ultimately activated, that aids in $\mathrm{T}$ synthesis as well as aromatization to E2, and cause the hyperestrogenism status of the eutopic endometrium. E2 causes its proliferation as well as healing actions via E2 receptors (ER, ER $\beta$ in this case). Nevertheless in normal healing, escalated synthesis of E2 ends, but in the uterus they stimulate oxytocin modulated hyperperistalsis via $\mathrm{ER} \alpha$, that

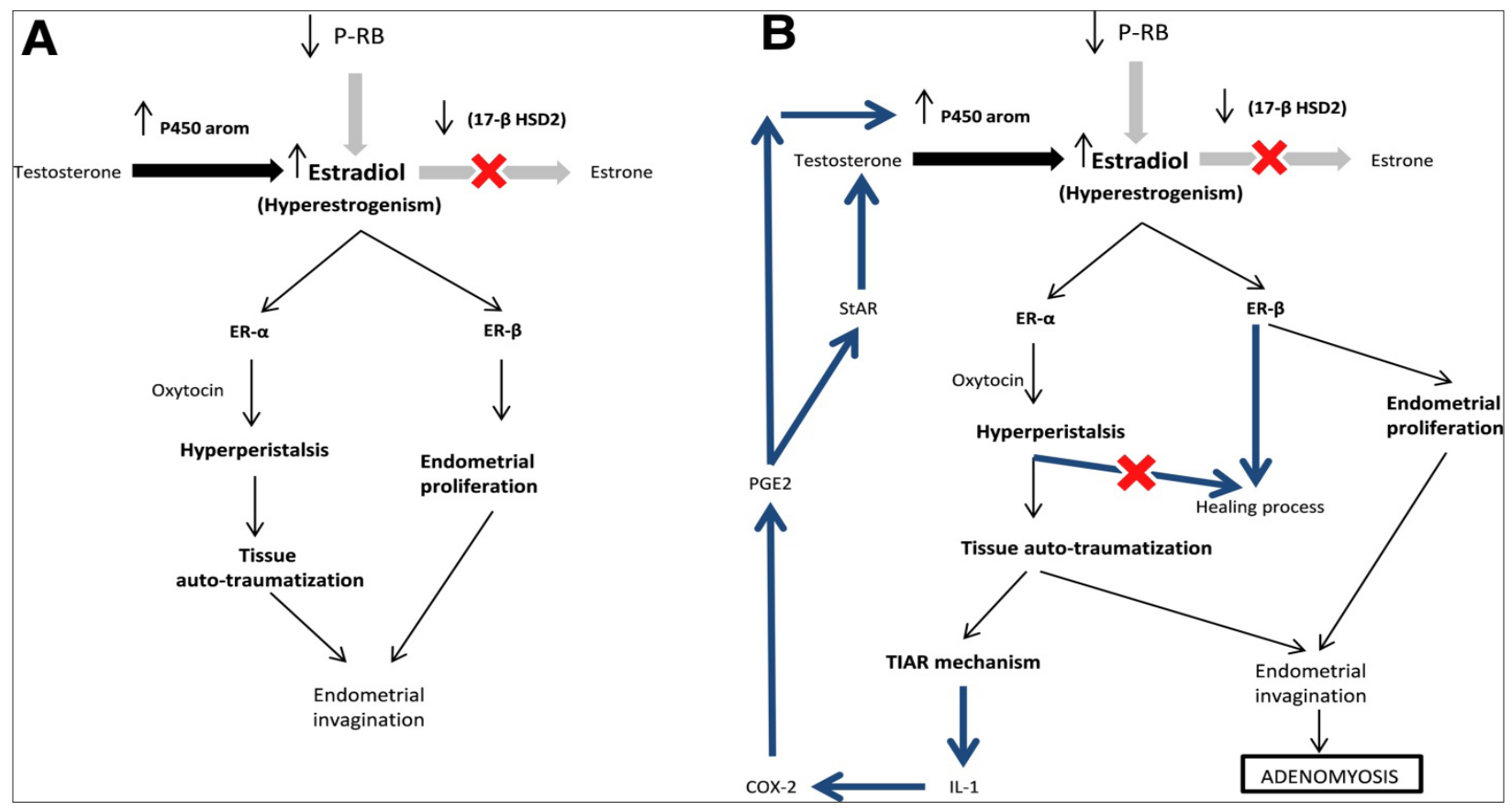

Figure1. Courtesy ref no-27-Invagination of the endometrial basalis: hyperestrogenism, hyperperistalsis, and TIAR mechanism activation. (A) A hyperestrogenic condition in the eutopic uterus may lead to increased proliferation in the endometrial basalis and tissue microtrauma in the vicinity of the JZ, thus allowing endometrial intramyometrial invagination. (B) As a consequence of tissue microtrauma, the TIAR mechanism is activated (blue arrows), generating a mechanism of positive feedback whereby estrogen production promotes uterine peristalsis and further autotraumatization, progressively worsening the microtrauma and endometrial invagination and eventually leading to adenomyosis establishment. inhibits the healing process (fig1B). Hence a positive feedback mechanism gets developed, via which chronic hyperperistalsis in the JZ facilitates repeated cycles of autotraumatiization, causing a continuous disruption of the muscular fibers in the myometrial wall. This keeps on worsening with every cycle and ultimately escalates invagination of the endometrial basal layer into the myometrium, ultimately ending in the formation of adenomyotic lesions [fig1A, 2A awa 2a). Further as the expression of matrix metallo proteinases (MMPs) -2 as well as 9 were observed to be significantly greater in the eutopic endometrium of the adenomyotic lesions, which in the endometrium of the disease free women [29], it is probable that the proteasesmightalso be involved in the intra myometrial endometrial invagination process. Additionally, since they might present with tissue damage to the escalated birth rates and prior uterine surgery were shown in a lot of retrospective studies to be risk factors for adenomyosis formation [30-32], that further reinforces the critical significance of tissue microtrauma in the formation of this disease. endometrial myometrial interface, caesarean delivery, 
An Update on the Various Theories of the Origin of Uterine Adenomyosis -A Systematic Review

Metaplasia of Displaced Embryonic Pluripotent Mullerian Remnants leading to Differention of Adult Stem Cells

Though a lot of human as well as experimental studies prefer the posit of endo myometrial invagination, with the current insight of adenomyotic lesions might occur de novo via metaplasia of the displaced embryonic pluripotent Mullerian Remnants or actually via differention of Adult Stem Cells [33-35] .

The Mullerian ducts represent primordial embryological structure which at the time of fetal life, form the female uterine tract (uterus, fallopian tubes, as well as the superior part of the vagina)[36]. These ducts comprise of surface epithelium as well as the underlying urogenital ridge mesenchyme with the ability to differentiate into endometrial glands and stroma [37]. It was posited that metaplastic alterations of intra myometrial Embryonic mullerian remnants in the adult uterine wall can probably result in development of denovo ectopic endometrial tissue within the myometrial wall, forming adenomyotic lesions [35] (fig2B). On the same line since their histology shows classical features of adenomyosis with smooth muscle hyperplasia as well as fibrosis, deep endometriotic nodules were also pointed to be probably secondary to mullerian rest differention, atleast in certain subjects or due to the adenomyotic tumoral lesions initiating from the cervix as pointed by Donnez et al, about 20 yrs back [38-41]. This same mode might explain how ectopic endometrial cells might penetrate the uterine wall as well as develop a form of uterine adenomyotic nodule $[39,40]$ which might continue posteriorly in the direction of the external wall of the rectum or the posterior part of cervix, and form a cervical adenomyotic nodule which might extend posteriorly in the direction of the external wall of rectum. Deep lesions are basically separate from peritoneal endometriosis [38,41], as well as their histology is quiet similar to that of uterine adenomyosis. The mullerian metaplasia theory gets corroborated further by case reports of confirmed adenomyosis in the rudimentary muscular uterine wall of Rokitansy -Kuster-Hauser syndrome with lack of functional endometrium [42-44].

Whereas ectopic mullerian remnants documented in both epithelial as well as stromal Adult Stem Cells having clonogenic action were found in colony forming units of endometrial as well as stromal cells derived via hysterectomy specimens [45]. It is believed that these Stem Cells remain in the endometri al basalis following menstruation [46.47]. In case of healthy endometrium, the location of Stem Cells in the endometrial basalis is a key factor for regeneration as well as replacement, but it might let uninhibited growth which might reach beyond the endometrium [48] Adult progenitor Stem Cells might get deposited in the uterus following retrograde menstruation and then differentiate into endometrial glands as well as stroma. These might then form into denovo intra myometrial implants or along with menstrual debris, develop an endometrial colony, thus forming focal uterine adenomyosis (fig2C). Though it is still not clear if endometrial fragments are shed via basalis during menstruation, in case of similar uterine abnormalities like endometriosis, escalated rates of basalis shedding were documented as compared to disease free women [49]. For analysis of potential part retrograde menstruation in endometrial Stem Cells implantation in the uterine wall further studies are needed for knowing if same menstrual patterns are seen in cases of adenomyosis .

On the other hand Vannucinini et al. as well as Gargett et al. posited that endometrial Stem Cells might get activated following tissue damage [33,34]. Thus, tissue microtrauma to the JZ as well as endometrial basalis might result in abnormal changes of the Stem Cell niche, letting their differentiating progeny to migrate towards the myometrium instead of endometrial functionalis probably causing adenomyosis formation [33,34](fig3).

Recently following electron microscopy evaluation, Ibrahim et al. detailed another Stem Cell-like population in the endometrial-myometrial interface of the fundocornual raphe [50]. These Stem Cells are presenteccentricallyin the epithelial glands of the basal endometrium as well as in view of electron-lucent look of their cytoplasm they were labeled pale cells [50]. In the endometrial-myometrial interface of adenomyotic women, same Stem Cells were significantly $<$ attached to their surrounding epithelial cells (absence of desmosomal junctions) and displayed $>$ pseudopods, as compared to what was seen in disease -free women. Thus it was pointed that adenomyosis, pale cells might get translocated via the basal membrane of the adenomyotic glands, develop motile properties and move toward the stromal compartment and then in the myometrium, where they can form de novo adenomyotic lesions [50]. This migration might get escalated by the enhanced expression of matrix 
An Update on the Various Theories of the Origin of Uterine Adenomyosis -A Systematic Review

metalloproteinase(MMP)2 as well as 9 in the stromal cells in the eutopic endometrium of patients with in vivo adenomyosis $[29,51]$.

Thus even if there is robust proof to corroborate the invagination theory, the de novo formation of adenomyotic lesions is further a probability which can't be given up. Greater researchers are required for establishing a part of mullerian remnants as well as endometrial Stem Cells in the starting of adenomyosis[52].

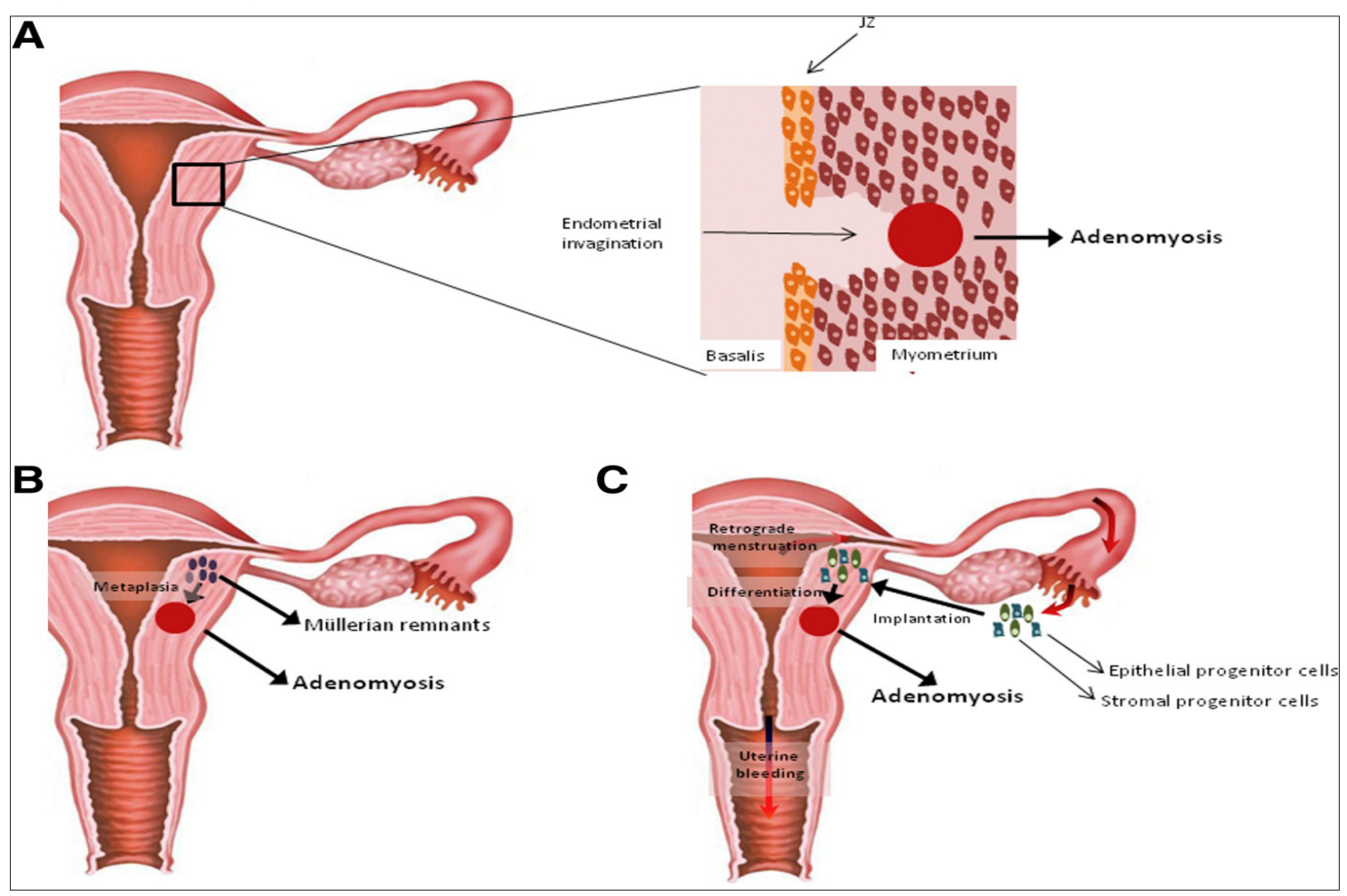

Figure2. Courtesy ref no-27-Theories on the origin of adenomyosis. (A) Invagination of the endometrial basalis into the myometrium, after TIAR mechanism activation. $(B, C)$ De novo formation of lesions: (B) after metaplasia of displaced embryonic pluripotent remnants or $(C)$ from differentiation of endometrial and stromal stem cells deposited in the myometrium after retrograde menstruation.

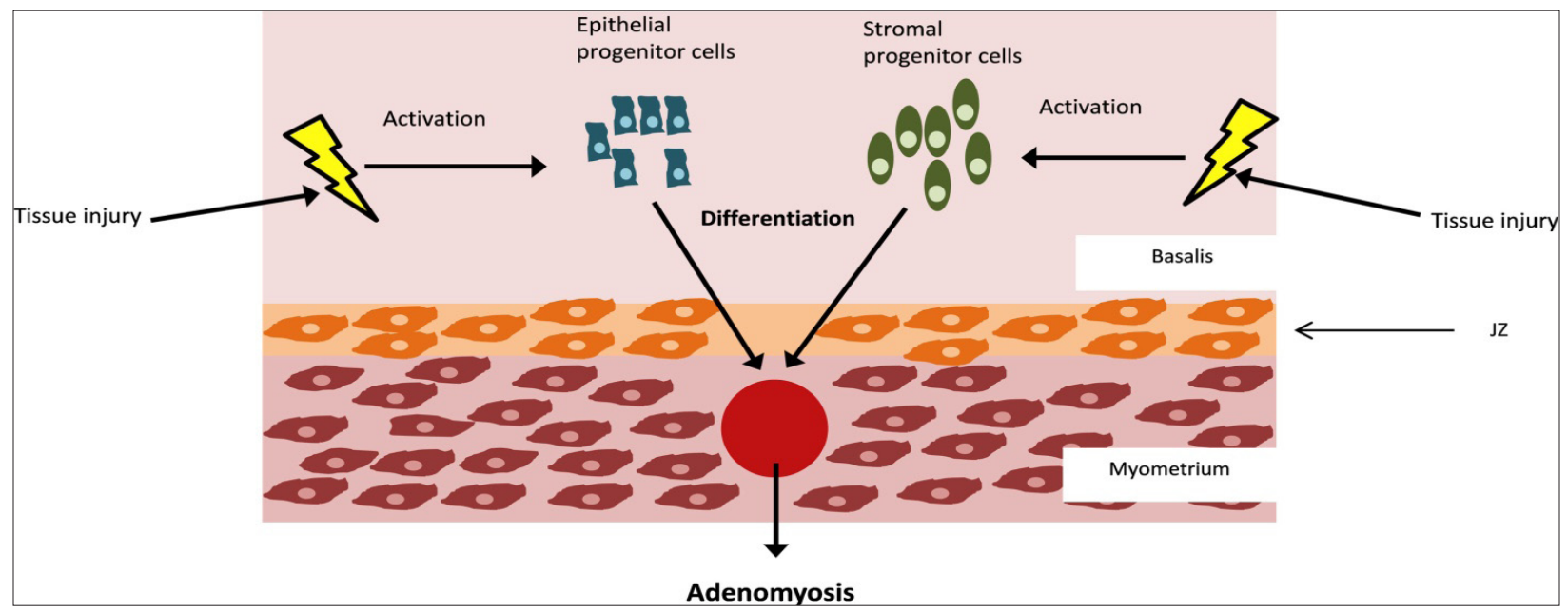

Figure3. Courtesy ref no-27-De novo formation of adenomyotic lesions from endometrial and stromal stem cells after tissue injury. Tissue microtrauma to the JZ and endometrial basalis may lead to abnormal alteration of the stem cell niche, allowing their differentiating progeny to move toward the myometrium rather than the endometrial functionalis, possibly resulting in adenomyosis establishment. 
An Update on the Various Theories of the Origin of Uterine Adenomyosis -A Systematic Review

Pathogenic Development

Once adenomyosis gets set in, migration as well as invasion are thought to be critical factors in the progress as well as spread of lesions. EMT represents a biological function that is implicated in embryological formation as well as tissue repair as well as cancer cell migration, where stationary polarized epithelial cells lose their cell-cell adhesions as well as convert it to markedly motile mesenchymal cells, increasing their migratory as well as invasive abilities [53]. Thus functional loss of expression of the cell -cell adhesion marker E-cadherin in epithelial cells as well as simultaneous escalated expression of the mesenchymal markers $\mathrm{N}$ cadherin expression as well as vimentin were found as a hall mark of Epithelial-mesenchymal transition (EMT)[54]. In case of adenomyotic lesions reduced E-cadherin expression as well as escalated expression of vimentin as well as $\mathrm{N}$ - cadherin were found in the epithelial cells of adenomyotic lesions as compared to normal endometrium (controls) [55], pointing that a process which corroborates and facilitates invasion as well as propagation of adenomyotic lesions might actually get dictated via EMT.

The mode that triggers EMT in adenomyosis has not been yet demonstrated. Nevertheless, in consistency with the theory of invagination as well as hyperestrogenic status of adenomyotic lesions, Chen et al. gave proof of the estrogen dependency of EMT activation in this uterine condition [55], with estrogens, ER-positive Ishikawa endometrial epithelial cells expressed great amounts of Slug, a transcription factor which regulates EMT via repression of E-cadherin [56], along with the mesenchymal muscle markers $\mathrm{N}$ - cadherin expression as well as vimentin than did controls [55]. These authors further demonstrated that following treatment with raloxifene, a selective estrogen receptor modulator which blocks estrogen stimulated signaling [57], the mesenchymal phenotype gets reverted, pointing that raloxifene might be an efficacious treatment to prevent EMT -induced propagation of adenomyotic lesions .

Ontheotherhand,plateletcollectionaswellasactivation were also documented to be a probable etiology of
EMT -induction in adenomyosis. Possibly in view of activation of the TIAR mode, platelets were observed to be markedly aggregated in the pericellular spaces of vimentin positive stromal cells of adenomyotic lesions [58]. Shen et al. studying adenomyosis in mice found escalated platelet aggregation as well as significantly escalated immunostaining of transforming growth factor beta (TGF $\beta$ ), p-Smad 3 as well as vimentin in the ectopic endometrium of mice with adenomyosis as the disease propagated, whereas immunoreactivity for E-cadherin kept on reducing at a steady pace [59]. Subsequent studies conducted in mice showed that antiplatelet therapy actually has a potential for treatment angle [60]. Actually Liu et al. demonstrated platelet aggregation to be associated with the initiation of EMT via the activation of TGF $\beta 1 /$ Smad 3 signaling pathway in human adenomyosis [58].

Conversely, in a baboon model, that got formed more recently [15], lesions accumulated $1 \mathrm{yr}$ following adenomyosis induction demonstrated no significant variations in the amounts of the epithelial cell-cell adhesion markers, E-cadherin as well as $\beta$-catenin among the maximum aggressive as well as the least aggressive adenomyotic glands [61]. As per Friedl et al. sustainance of cell -cell attachment is the major property of collective cell migration, a process that involves massively coordinated migration of groups of cells involved in physiological(wound healing) as well as pathological (cancer) process[62], the reason Donnez et al. posited the role of this phenomenon in the invasiveness of this disease[16,61]. Nevertheless $6 \mathrm{mths}$ following adenomyosis stimulation, invasive lesions showed <levels of E-cadherin as well as $\beta$-catenin as compared to noninvasive lesions [16]. These outcomes associate with the decrease, E-cadherin seen by Shen et al. following 60days in adenomyosis stimulated mouse model[59], pointing that EMT might be responsible for early disease propagation, whereas collective cell migration, is the predominant phenomena in later invasive processes in adenomyosis.

Without the mode of invasion, the cell migration event always needs breakdown as well as reconstruction of the extracellular matrix (ECM). Chen etal. very recently 
An Update on the Various Theories of the Origin of Uterine Adenomyosis -A Systematic Review

demonstrated an overexpression of the nuclear transcription factor Nrf2 (erythroid -E2 -related factor 2) in the glandular epithelium of adenomyotic lesions as compared to disease free women [63]. They showed that Nrf2 overexpression has a direct association with an escalated expression of MMP9 that has a significant part in ECM degradation [63]. Parallel with this proposal, expression of MMPs-2 as well as 9, is markedly >in adenomyotic lesions as compared to normal endometrium of healthy women [29]. Further a >risk of adenomyosis was documented in women carrying MMP-1-16071 G/2G as well as MMP-21306 C/T Polymorphisms in their promoter area $[64,65]$. These observations might corroborate that overexpression of the nuclear transcription factor Nrf2 might trigger intramyometrial migration of endometrial implants, via the control of MMP9 as well as another MMP, hence implicates Nrf2 as well as a MMP in the pathogenic evolution of adenomyosis.

From the above details, it is understood that for facilitating their invasiveness across the myometrium, adenomyotic cells acquire migration characteristics like absence of cell-cell attachment or the ability of degrading the ECM. Moreover, the presence of intravascular adenomyotic tissue, was documented in the myometrium of adenomyotic patients, hence it is believed that in certain cases, the vascular network might also aid in the local migration as well as spread of these lesions across the uterine wall[66].

\section{RISK FACTORS}

\section{Clinical History of Patients}

Risk Factors relate to adenomyosis are early menarche, short menstrual cycles, escalated body mass index(BMI), as well as history of depression[31]. As described earlier, surgical tissue damage to the endometrial -myometrial interface is a Risk Factors for adenomyosis [30-32].

It is not clear if smoking is a risk factor for adenomyosis. Lower rates of adenomyosis was documented by Parazzini et al. [30] as compared to women who had never smoked, but other studies demonstrated either
$>$ rates of smoking in adenomyotic patients [67] or no correlation among these 2 factors[68].

Proof regarding correlation among adenomyosis as well as estrogen exposure is also controversial. Actually whereas Parazzini et al. [30] could not find any correlation between the risk of adenomyosis as well as utilization of oral contraceptives (OC's) . This association still needs future evaluation further, since OC's are a commonly utilized therapy for dysmenorrhea as well as heavy menstrual bleeding, that are common symptoms of patients with adenomyosis[31].

A > prevalence of adenomyosis has been published regarding women who were given tamoxifen for the therapy of breast cancer [69-71]. Tamoxifen is a nonsteroidal antiestrogen commonly utilized for treatment in postmenopausal breast carcinoma subjects. Despite blocking ER's in breast tissue, in other tissues like endometrium, it might be having an estrogenic action as well as facilitate proliferation as well as adenomyosis formation or reactivation of preexisting adenomyotic lesions.

\section{Genetic Predisposition}

Gene deletions might also enhance the susceptibility of adenomyosis. Earlier it had been documented that exposure to endocrine disruptive chemicals, like phthalates, is correlated with a >chance of adenomyosis[72]. Actually escalated prevalence of this uterine problem was seen in patients who carried null genetic variant for the detoxification enzyme glutathione $S$ transferased $\mu 1$ (GSTM1) as well as showing escalated levels of urinary mono-ethyl hexyl phthalate $[73,74]$. This suggests a probable additive actions of these estrogenic chemical as well as gene deletions, giving rise to adenomyosis formation.

\section{Epigenetic Changes}

Epigenetic Changes have been found in adenomyosis. deoxyribonucleic acid (DNA) methyl transferases (DNMTs) represent a family of enzymes which catalyze the transfer of a methyl group to DNA, Causing a $>$ compact chromatin and thus gene repression[75]. Escalated expression of DNMT1 as well as DNMT3B was reported as compared to controls [76]. Parallel to 
An Update on the Various Theories of the Origin of Uterine Adenomyosis -A Systematic Review

these observations, hypermethylation of the promoter region of PRB was found in stromal cells of adenomyotic nodules, resulting in silencing of, that agreed with the previous mode of P resistance [24]. Further aberrant expression of histone deacetylases (HDACs) was also observed in women having adenomyosis. Actually, immunoreactivity of HDAC1 as well as HDAC 3 was increased in ectopic as well as eutopic endometrium of adenomyotic lesions as compared to controls [77]. This points the role of histone modifications in the etiopathogenesis of adenomyosis. Hence therapy with valproic acid, that is an HDAC inhibitor, caused amelioration of dysmenorrhea as well as hyperalgesia as well as retardation of myometrial infiltration in patients presenting with adenomyosis[24, 78-80].

Together these observations seems to point that adenomyosis just like endometriosis might be an Epigenetic disease [81, 82].

Further Kobayashi et al. focusing on etiopathogenesis of Adenomyosis is a common gynecologic disorder defined by the presence of endometrial glands and stroma within the uterine myometrium. This review focusses on: (1) current understanding of cellular and molecular mechanisms of adenomyosis-related fibrogenesis, (2) transforming growth factor beta (TGF- $\beta$ )-dependent or TGF- $\beta$-independent mediators of fibrogenesis, and (3) the origin of fibrogenic myofibroblasts. They collected a literature search from PubMed and EMBASE database up to December 2018. First, causative factors of adenomyosis were classified into exogenous traumatic damage (surgical interventions, including curettage, normal delivery, or cesarean section) and endogenous traumatic damage (mechanical strain or myometrial hyperperistalsis). The mechanical forces and injury (microdehiscences) are fundamental regulators of cell behavior and central to our understanding of disease pathogenesis. Adenomyosis is characterized by abnormal response to injury and activation of myofibroblasts in the myometrium through altered barrier function of the endometrial-myometrial junctional zone (EMJZ). Second, they summarize recent advances on the molecular mechanism of fibrosis. Two distinct populations of myofibroblasts, highly myogenic cells, and nonmyogenic cells arise possibly through the TGF$\beta$-dependent and TGF- $\beta$-independent processes. TGF$\beta$-independent mechanisms are still intriguing and far from clear. Third, the importance and implications of resident fibroblasts, bone-marrow stem cells-derived fibrocytes, and epithelial-mesenchymal transitionderived myofibroblasts in fibrosis remain uncertain. Finally, originally adenomyosis was believed to be the single entity, but this disorder is composed of multiple heterogeneous subtypes. Keymediators of fibrogenesis may vary widely and largely depend on adenomyosis subtype. In conclusion, both cyclic mechanical strain and EMJZ weakness (microdehiscences) may be a prerequisite for adenomyosis fibrogenesis through the mechanotransduction process. Since there are significant molecular variations among affected individuals, the approach to identify key mediators of fibrosis remains challenging [83]. Further Guo SW reveiewed beyond TIAR aswell as invaginatiom In addition, two prevailing theories, i.e., invagination-based on tissue injury and repair (TIAR) hypothesis-and metaplasia, on adenomyosis pathogenesis, are briefly overviewed and then critically scrutinized. Both theories have apparent limitations, i.e., difficulty in falsification, explaining existing data, and making useful predictions. Based on the current understanding of wound healing, a new hypothesis, called endometrial-myometrial interface disruption (EMID), is proposed to account for adenomyosis resulting from iatrogenic trauma to EMI. The EMID hypothesis not only highlights the more salient feature, i.e., hypoxia, at the wounding site, but also incorporates epithelial mesenchymal transition, recruitment of bone-marrow-derived stem cells, and enhanced survival and dissemination of endometrial cells dispersed and displaced due to iatrogenic procedures. More importantly, the EMID hypothesis predicts that the risk of adenomyosis can be reduced if certain perioperative interventions are performed. Consequently, from a pathogenic standpoint, adenomyosis is not simply endometriosis of the uterus, and, as such, may call for interventional procedures that are somewhat different from those for endometriosis to achieve the best results [fig4-6]. 
An Update on the Various Theories of the Origin of Uterine Adenomyosis -A Systematic Review

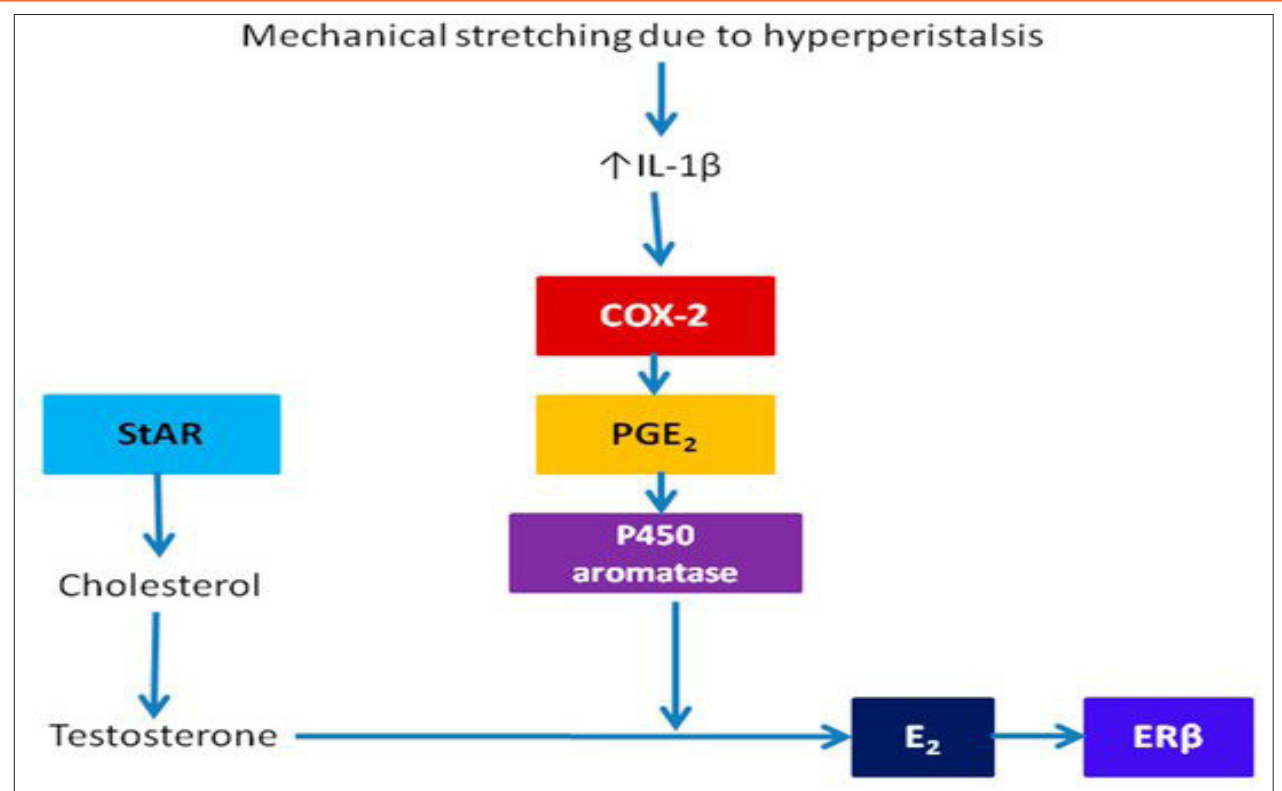

Figure4. Courtesy ref no -84. The key molecular signaling events initiated by the tissue injury and repair (TIAR) that leads to the increased local production of estradiol, as proposed by Leyendecker et al. [25,26]. Gene/protein names: COX-2: cyclooxygenase-2; E 2 : 17 $\beta$-estradial; ER $;$ : estrogen receptor $\beta$; IL-1 $\beta$ : interleukin-1 $\beta$; P450 aromatase: aromatase; PGE 2 : prostaglandin E2; StAR: steroidogenic acute regulatory protein.

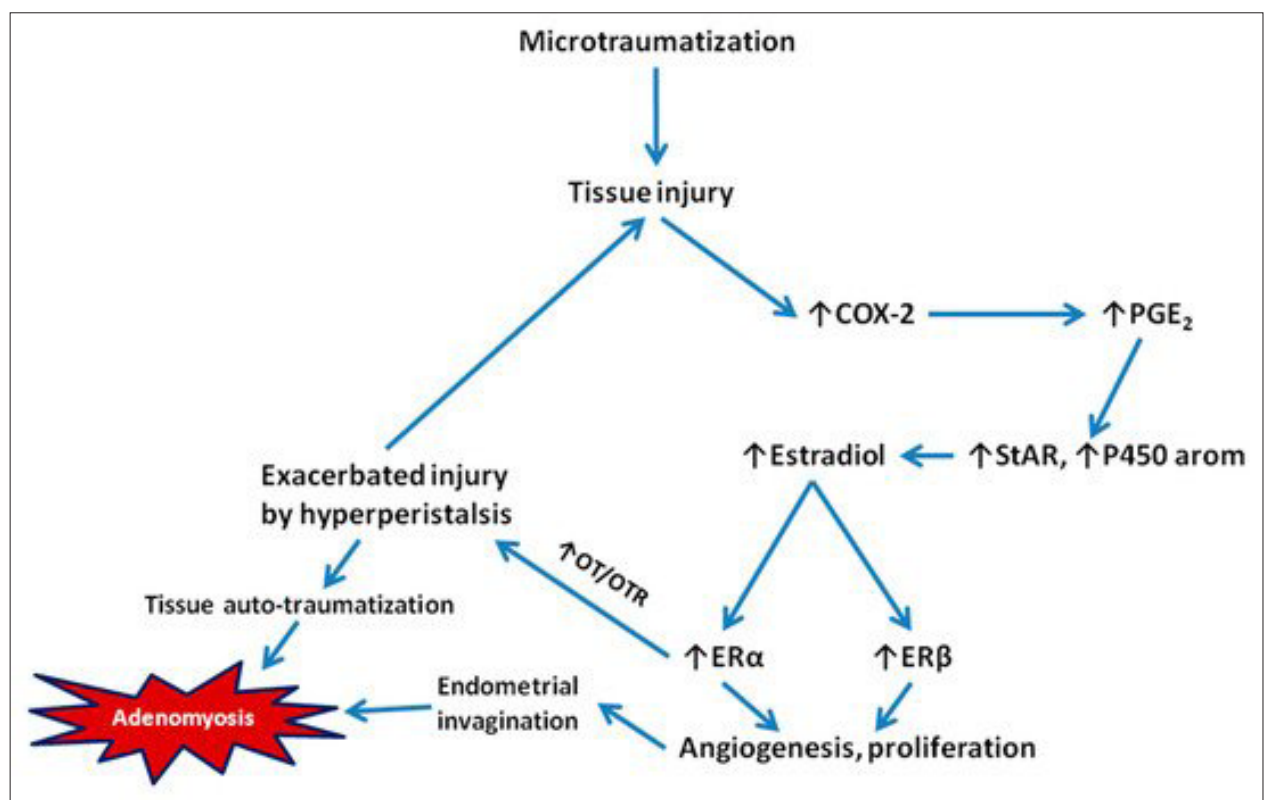

Figure5. Courtesy ref no-84-Leyendecker's model of tissue injury and repair (TIAR) that initiates the genesis of adenomyotic lesions [25,26]. Briefly, microtraumatization in the endometrial-myometrial interface causes tissue injury, which subsequently induces upregulation of COX-2 and increased production of PGE 2 , which, in turn, induces the expression of genes critical to estrogen production such as StAR and aromatase, resulting in increased local estrogen production. The elevated estrogen levels would activate both ER and ER $\beta$, leading to the induction of the OT/OTR signaling and subsequent increased uterine peristalsis and increased angiogenesis and proliferation. The increased peristalsis would further exacerbate uterine hyperperistalsis and thus TIAR, causing endometrial invagination and ultimately the formation of adenomyotic lesions. Gene/protein names: COX-2: cyclooxygenase-2; E 2 : $17 \beta$-estradial;

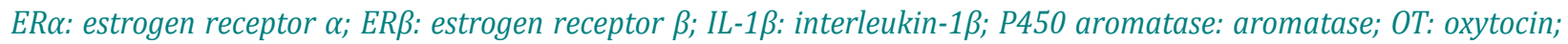
OTR: oxytocin receptor; PGE 2 : prostaglandin E2; StAR: steroidogenic acute regulatory protein. 
An Update on the Various Theories of the Origin of Uterine Adenomyosis -A Systematic Review

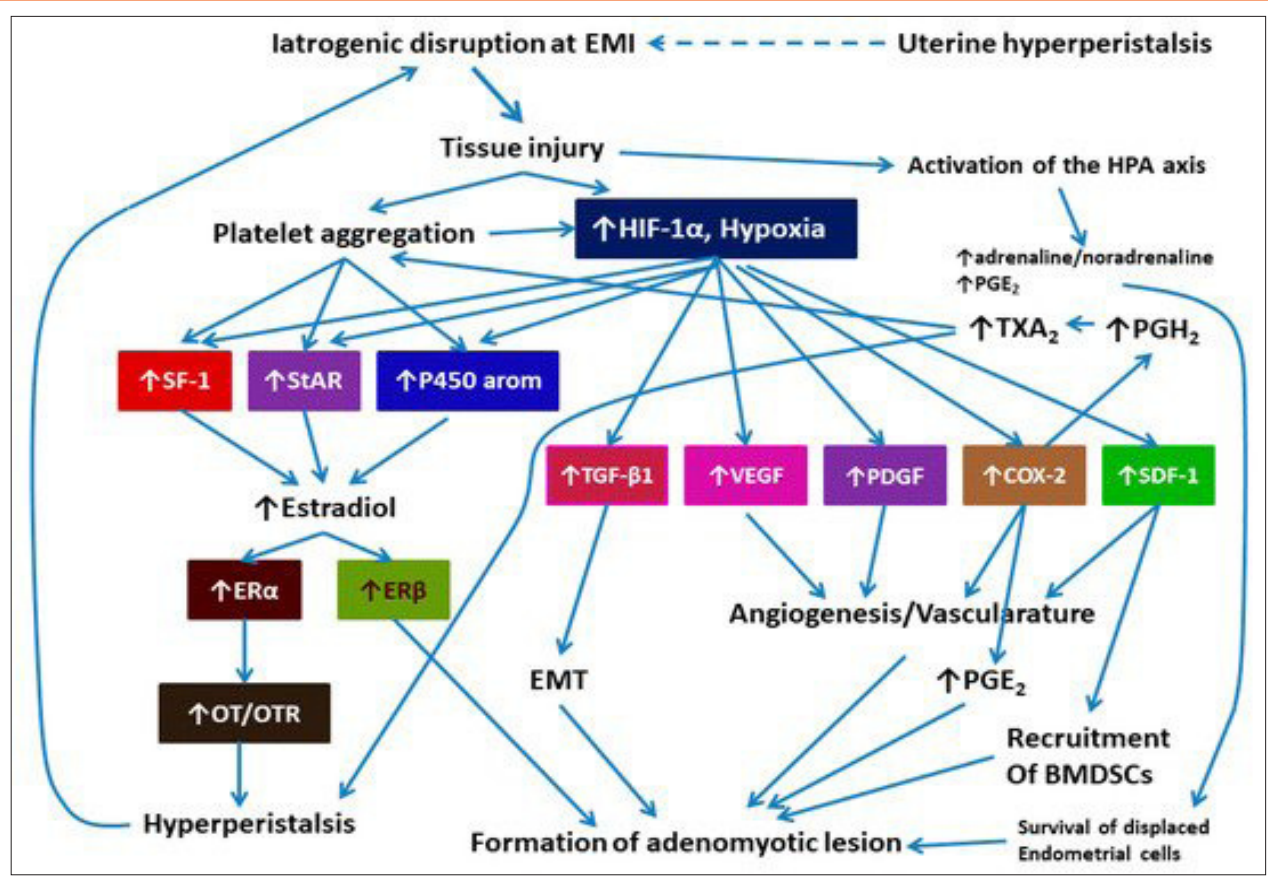

Figure6. Courtesy ref no-84-Schematic illustration of the formation of adenomyotic lesions due to the endometrialmyometrial interface disruption (EMID). Iatrogenic procedures causes disruption at the endometrial-myometrial

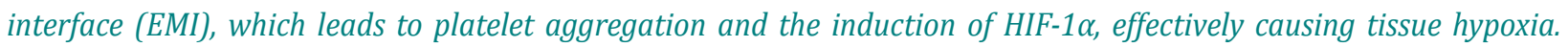
Uterine hyperperistalsis may also induce EMI disruption (shown in dashed arrow). As a result, genes involved in estrogen production are upregulated, resulting in increased local production of estrogen and subsequent induction of both ER $\alpha$ and ER $\beta$, which, in turn, leads to the induction of the OT/OTR signaling and increased uterine peristalsis. In addition, tissue hypoxia activates TGF- $\beta 1, V E G F, P D G F, C O X-2$, and SDF-1 signaling pathways, leading to increased angiogenesis, vascularature, and the recruitment of BMDSCs to the wounding site. The induction of COX-2 would also increase the production of PGH 2 and TXA 2 , which also enhances uterine peristalsis. Moreover, the TGF- $\beta 1$ signaling pathway induces EMT, leading to the invasion of endometrial epithelial cells to the EMI and further down to the myometrium. Tissue injury also would activate the HPA axis, leading to the release of catecholamines and PGE 2 , which collectively result in impaired cell-mediated immunity and, as such, enhances the survival of displaced and dispersed endometrial cells within the myometrium. All these events ultimately lead to the formation of adenomyotic lesions in the myometrium. Abbreviations used: BMDSC: bone marrow derived stem cells; COX-2: cyclooxygenase-2; E 2: 17 $\beta$-estradial; EMI: endometrial- myometrial interface; EMT: epithelial-mesenchymal transition; ER $\alpha$ : estrogen receptor $\alpha$; ER $\beta$ : estrogen receptor $\beta$; HIF-1 $\alpha$ : hypoxia-inducible factor 1 $\alpha$; HPA: hypothalamic-pituitary-adrenal; P450 aromatase: aromatase; PDGF: platelet-derived growth factor; OT: oxytocin; OTR: oxytocin receptor; PGE 2: prostaglandin E2; PGH 2: prostaglandin H2; SDF-1: stromal cell-derived factor 1; SF-1: steroidogenic factor-1; StAR: steroidogenic acute regulatory protein; TGF-

B1: transforming growth factor $\beta 1$; TXA 2 : thromboxane A2; VEGF: vascular endothelial growth factor.

\section{CONCLUSIONS}

Adenomyosis that gets diagnosed frequently is an estrogen dependent gynaecological problem which presents with pelvic pain, AUB, as well as infertility. There are basically 2 theories posited for the origin of adenomyosis. The commonest believed is the role of tissue injury as well as repair mode and probably claims which point adenomyosis occurs secondary to invagination of the endometrial basalis into the myometrium. Another theory says that adenomyotic lesions occur via metaplasia of displaced embryonic pluripotent mullerian remnants or differentiati on of adult stem cells. Earlier evaluation was done in human adenomyotic lesions as well as confirmed via evaluation in mice as well as baboons, that corroborated the role of EMT process in the early stages of propagation as well as spread of adenomyosis as well as suggested that collective cell migration might be involved in the later stages of invasion. Further. Based on the current understanding of wound healing, a new hypothesis, called endometrial-myometrial 
An Update on the Various Theories of the Origin of Uterine Adenomyosis -A Systematic Review

interface disruption (EMID), is proposed to account for adenomyosis resulting from iatrogenic trauma to EMI. The EMID hypothesis not only highlights the more salient feature, i.e., hypoxia, at the wounding site, but also incorporates epithelial mesenchymal transition, recruitment of bone-marrow-derived stem cells, and enhanced survival and dissemination of endometrial cells dispersed and displaced due to iatrogenic procedures. More importantly, the EMID hypothesis predicts that the risk of adenomyosis can be reduced if certain perioperative interventions are performed. Consequently, from a pathogenic standpoint, adenomyosis is not simply endometriosis of the uterus, and, as such, may call for interventional procedures that are somewhat different from those for endometriosis to achieve the best results.

\section{REFERENCES}

[1] Devlegher R, D'Hooghe T, TimmermanD. Uterine Adenomyosis in the infertility clinic. Hum Reprod Update 2003;9:139-47.

[2] Siegler AM, Camilien L. Adenomyosis. J Reprod Med 1994;39:841-53.

[3] Van den Bogh T, Dueholm M, Leone EPG, Valentin L, Rasmussen CK, Votino A, et al. Terms, definitions and measurements to describe sonographic features of myometrium and uterine masses :A consensus opinion from the Morphological Uterine Sonographic Assessment (MUSA)group . Ultrasound Obstet Gynaecol 2015;46:284-98.

[4] Chapron C, Tosti C, Marcellini L, Bourdon M, Lafay-Pillet MC, Millischer AE, et al. Relationship between the magnetic resonance imaging appearance of Adenomyosis and endometriosis phenotypes. Hum Reprod 2017;32:1393-401.

[5] Guo SW, Mao X, Ma Q, Liu X. Dysmenorrhea and its severity are associated with increased uterine contractility and overexpression of oxytocin receptor (OTR):in women with asymptomatic Adenomyosis. Fertil Steril 2013;99:231-40.

[6] Vercellini P, Consonni D, Dridi D, Bracco B, Frataruolo MP, Somigliana E. Uterine Adenomyosis and in vitro fertilization outcome :a systematic review and meta-analysis. Hum Reprod 2014;29:473-9.

[7] Lazzeri L, Govvani A, Exacoutos C, Tosti C, Prizauti S, Matzoni M, et al. Preoperative and post operative clinical and transvaginal Ultrasound findings of Adenomyosis in patients with deep infiltrating endometriosis. Reprod Sci 2014;21:1027-33.

[8] Gordts S, Grimbizis G, Campo R. Symptoms and classification of uterine Adenomyosis, including the place of hysteroscopy in the diagnosis. Fertil Steril 2018;109(3):380-8.

[9] Liu X, YuS, Guo SW. A pilot study on the use of Androgro pholide to treat symptomatic Adenomyosis. Gynecol Min Invas Ther 2014;3:119-26.

[10] Thain S, Tan HH. Approaches of adenomyomectomy. Gynecol Min Invas Ther 2015;4:49-51.

[11] Streuli I, Dubuisson J, Santulli P, De Ziegler D, Batteux F, Chapron C. An update on the pharmacological management of adenomyosis . Expert Opin Pharmacother 2014;15:2437-60.

[12] Bazot M, Darai E. Role of trans vaginal sonography and magnetic resonance imaging in the diagnosis of adenomyosis. Fertil Steril 2018;109(3):38997.

[13] Leydenchecker G, Bilgicyildirim A, Inacket A, Staff T, Huppert P, Mall G, et al. Adenomyosis and endometiosis:Revisiting their association and further insights into the mechanisms of autotraumatization:An MRI study . Arch Gynaecol Obstet 2015;291:917-32.

[14] Li B, Chen M, Liu X, Guo SW. Constitutive and tumor necrosis factor alpha induced activation of nuclear factor kappa B in Adenomyosis and its inhibitor of Androgro pholide. Fertil Steril 2013;100:568-77.

[15] Donnez O, Van Langendonrickt A, Defrete S, Colette S, Van Kerk O, Dehoux JP, et al. Induction of endometiotic nodules in an experimental baboon model mimicking human deep nodular lesions. Fertil Steril 2013;99:783-9. e3.

[16] Donnez O, Orellana R, Van Kerk O, Dehoux JP, Donnez J, Dolmans MM. Invasion process of deep nodular endometiosis in an experimental baboon model:similarities with collective cell migration? Fertil Steril 2015;104:1-7. e2.

[17] Benagliono G, Brosens I, Habiba M. Structural 
An Update on the Various Theories of the Origin of Uterine Adenomyosis -A Systematic Review

and molecular features of the endomyometrium in endometriosis and Adenomyosis. Hum Reprod Update 2014;20:386-402.

[18] Rizner TL. The important role of steroid sulfatase and sulfotransferase in gynaecological disease . Front Pharmacol 2016;7:30.

[19] Wang Y, Qu Y, Song W. Genetic variant in COX2-1195 and the risk of endometriosis and Adenomyosis. Clin Expert Obstet Gynaecol 2015;42:168-72.

[20] Tong X, Li Z, Wu Y, Fu X, Zhang Y, Fan H. COMT 158G/A and CYP1B1 432 C/G polymorphisms increase the risk of endometriosis and Adenomyosis. Eur J Obstet Gynaecol Reprod Biol 2014;179:17-21.

[21] Kitawaki J, Noguchi T, Amatsu T, Maeda K, Taukamoto K, Yamamoto T, et al. Expression of Aromatase cytochrome $\mathrm{P} 450$ protein and messenger ribonucleic acid in human endometriotic and Adenomyotic tissues but not in normal endometrium. Biol Reprod 1997;57:514-9.

[22] Kitawaki J, Koshiba H, Ishihara H, Kusul IJ, Tsukamoto K, Honjo H. Progesterone induction of of 17-ßhydroxy steroid dehydrogenase type2 during the secretory phase occurs in the Estrogen dependent benign diseases but not in normal endometrium. Biol Reprod 1997;57:514-9.

[23] Mehasseti MK, Pancrial R, Taylor AH, Brown I, Bell SC, Habiba M. Estrogen and progesterone receptor isoform distribution through the menstrual cycle in uteri with and without Adenomyosis. Fertil Steril 2011;95:2228-35.

[24] Jichan N, Xishi L, Guo SW. Promoter hypermethylation of progesterone receptor isoform B(PR-B) in Adenomyosis and its rectification by a histone deacetylase inhibitor and a demethylation agent. Repr Sci 2010;17:9951005.

[25] Leydenchecker G, Wildt L. A new concept of endometriosis and Adenomyosis: tissue injury and repair(TIAR). Horm Mol Biol Clin Invest 2011;5:125-42.

[26] Leydenchecker G, Wildt L, Mall G. The pathophysiology of endometriosis and
Adenomyosis: tissue injury and repair. Arch Gynaecol Obstet 2009;280:529-38.

[27] Garcia-Solares J, DonnezJ, Donnez O. Pathogenesis of uterine Adenomyosis:invagination or metaplasia. Fertil Steril 2018;109(3):371-9.

[28] Ibrahim MG, Siliem M, Plendl J, Chiantera V, Sehouli J, Mechsner S. Myofibroblasts are evidence of chronic tissue micriotrauma at the endometrial-myometrial junctional zone in uteri with Adenomyosis. Reprod Sci 2017;24:1410-8.

[29] Li T, Li YG, PuDM, . Matrix metallo proteinases 2 and 9 expression correlated with angiogenesis in human Adenomyosis. Obstet Gynaecol Invest 2006;62:229-35.

[30] Parazzini E, Vercellini P, Panazza S, Cgattenoud L, Oldani S, Crosignani FG. Risk factors for Adenomyosis. Hum Reprod 1997;12:1275-9.

[31] Templeman C, Marshall SF, Ursin G, Horn-Ross PL, Clarke CA, Allen M, et al. Adenomyosis and endometriosis in the California Teachers Study . Fertil Steril 2008;90:415-24.

[32] Riggs K, Lim EK, Liang D, Bullwinkel R. Caesarean section as a risk factor for the development of Adenomyosis uteri. JReprod Med 2014;59:20-4.

[33] VannucciniS, TostiC,CarmonaF,HuangSJ,Chapron C, Guo SW, et al. Pathogenesis of adenomyosis:an update on molecular mechanisms. Reprod Bimed Endocrinol 2017;35:592-604

[34] Gargett CE. Uterine stem cells :what is the evidence ? Hum Reprod Update2007;13:87-101.

[35] Ferenczy A. Pathophysiology of Adenomyosis. Hum Reprod Update1998;4:312-22.

[36] Sobel V, Zhu YS, Imperato-McGinley J. Fetal hormones and sexual differentiation. Obstet Gynaecol Clin North Am 2004;31:837-56.

[37] Spencer TE, Hayashi K, Hu J, Carpentier KD. Comparative developmental biology of mammalian uterus. Curr Top Dev Biol 2005;68:85-122.

[38] Donnez J, Nisolle M, Casena Roux E, Brion P, Da Costa Ferreira N. Steroidogenic evaluation of peritoneal endometriosis and endometriotic nodules in the rectovaginal septum. Hum Reprod 1996;11:224-8. 
An Update on the Various Theories of the Origin of Uterine Adenomyosis -A Systematic Review

[39] Donnez J, Nisolle M, Casena Roux E, Bassil S, Anaif V. Rectovaginal septum, endometriosis or Adenomyosis:laparoscopic management in a series of 231 patients. Hum Reprod 1995;10:630-5.

[40] Donnez J, Nisolle M, Gillerot S, Smrets M, Bassil S, Casena Roux E. Rectovaginal septum Adenomyotic nodules :a series of 500 cases. Br J Obstet Gynaecol 1997;104:1014-8.

[41] Nisolle M, Donnez J. Peritoneal endometriosis, and Adenomyotic nodules of Rectovaginal septum are three different entities. Fertil Steril1997;68:585-90.

[42] Enatsu A, Harada T, Yoshida S, Iwabe T, Terakawa N. Adenomyosis in a patent with Rokitansy -KusterHauser syndrome. Fertil Steril2000;73:862-3.

[43] Chun S, Kim YM, Ji YI. Uterine Adenomyosis which developed from hypoplastic uterus in postmenopausal woman with the with Rokitansy -Kuster-Hauser syndrome. J Menopausal Med 2013;19:135-8.

[44] Hoo PS. Norhaslinde AR, Reza JN. Rare case of leiomyoma and Adenomyosis in Mayer Rokitansy -Kuster-Hauser syndrome. Case Rep Obstet Gynaecol 2016;2016:3725043.

[45] Chan RW, Schwab KE, Gargett CE. Clonoigenicity of human endometrial epithelial and stromal cells . Biol Reprod 2004;70:1738-50.

[46] Gotte M, Wolf M, Staebler A, Buchweitz O, Kelsch $\mathrm{R}$, Schuring AN, et al. Increased expression of the adult Stem Cell marker Musashi-1 in endometriosis an d endometrial carcinoma. J Pathol 2008;215:317-29.

[47] Kato K. Stem Cells in human normal endometrium and endometrial carcinoma cells characterization of side population cells . Kahosiung J Med Sci 2012;28::63-71.

[48] Hullnagel d, Li F, Cosar E, Krikuri G, Taylor HS. The role of Stem Cells in the etiology and pathopathophyslogy of endometriosis. Semin Reprod Med 2015;33 :333-40.

[49] Leydenchecker G, Herbertz M, Kunz G, Mall G. Endometriosis results from the dislocation of basal endometrium. Hum Reprod 2002;17:272536.
[50] Ibrahim MG, Chiantera V, Frangini S, Younes S, Kohler C, Taube ET, et al. Ultramicrotrauma in the endometrial-myometrial junctional zone and pale cell migration in Adenomyosis. Fertil Steril2015;104:1475-83. e1-3.

[51] Yang JH, Wu MY, Chen MJ, Chen SU, Yang YS, Hu HN. Increased Matrix metallo proteinase 2 and tissue inhibitor of metallo proteinase 1 secretion but unaffected invasiveness of endometrial stromal cells in in Adenomyosis. Fertil Steril 2009;91(Suppl 2):2193-8.

[52] Gargett CE, Schwab KE, Deane JA. Endometrial stem/progenitor cells :the first 10yrs. Hum Reprod Update 2016;22:137-63.

[53] Voulgari A, Pintzas A. Epithelial-mesenchymal transition in cancer metastasis :mechanisms, markers and strategies to overcome drug resistance in the clinic. Biochim Biophys Acta 2009;1796:75-90.

[54] Polyol T, Weinberg RA. Transitions between Epithelial and mesenchymal states :acquisition of malignant and Stem Cell states :acquisition of malignant and Stem Cell traits . Nat Rev Cancer 200i9;9:265-73.

[55] Chen YI, Li HY, Huang CH, Twu NF, Yen MS, Wang $\mathrm{PH}$, et al. Oestrogen induced Epithelialmesenchymal transition of Endometrial Epithelial cells contributes to the development of Adenomyosis. J Pathol 2010;222:261-70.

[56] Nielo MA. The snail superfamily of zinc finger transcription factors. Nat Rev Mol Cell Biol 2002;3:155-66.

[57] Flamini MI, Sanchez AM, Goglia L, Tosi V. Genazzani AR. Simoncini T. Differential actions of Oestrogen and SERM's in regulation of the actin cytoskeleton of Endometrial cells. Mol Hum Reprod 2009;15:675-85.

[58] Liu X, Shen M, Qi O, Zhang H, Guo SW. Corroborating evidence for platelet induced Epithelial-mesenchymal transition and fibroblast-myo fibroblast trans Differentiation in the the development of Adenomyosis in mice. Hum Reprod 2016;31:734-49.

[59] Shen M, Liu X, Zhang H, Guo SW. Transforming growth factor beta signaling coincides with 
An Update on the Various Theories of the Origin of Uterine Adenomyosis -A Systematic Review

Epithelial-mesenchymal transition and fibroblast-myo fibroblast trans Differentiation in the the development of Adenomyosis in mice . Hum Reprod 2016;31:355-69.

[60] Zhu B, Chen Y, Shen X, Liu X, Guo SW. Antiplatelet therapy holds promises in treating Adenomyosis:experimental evidence. Reprod Biol Endocrinol 2016;14:66.

[61] Orellana R, Garcia-Solares J, Donnez J, Van Kerk O, Dolmans MM, Donnez O. Important role of collective cell migration and nerve fiber density in the development of deep nodular endometriosis. Fertil Steril 2017;107:987-95. e5.

[62] Friedl P, Locker J, Sahai E, Segali JE. Classifying collective cancer cell invasion. Nat Cell Biol 2012;14:777-83.

[63] Chen N, Du B, Zhou H, Shen F, Li J, Zie Z. Abnormal expression of Nrf2 may play an important role in the pathogenesis and the development of Adenomyosis. PLoS One 2017;12:e0182773.

[64] Kang S, Zhao X, Xing H, Wang N, Zhou R, Chen $\mathrm{S}$, et al. Polymorphisms in the Matrix metallo proteinase 2 and tissue inhibitor of metallo proteinase 2 and the risk of human Adenomyosis. Environ Mol Mutagen 2008;49:226-31.

[65] Ye H, He Y, Wang J, Song T, Lan Z, Zhao Y, et al. Effect of Matrix metallo proteinase2 promoter Polymorphisms on endometriosis, and Adenomyosis risk:evidence from a meta-analysis. J Genet 2016;95:611-9.

[66] Meenakshi M, McCluggage WG. Vascular involvement in Adenomyosis:report of a large series of common phenomena with observations on the pathogenesis of Adenomyosis. Int J Gynaecol Pathol 2019;29:117-21.

[67] Taran FA, Wallwiener M, Kabashi D, Rothmund R, Rall K, Kraemer B, et al. Clinical characteristics indicating Adenomyosis at the time of hysterectomy :a retrospective study in 291 patients. Arch Gynaecol Obstet 2012;285:1571-6.

[68] Parazzini E, Man V, Cipnani S, Busacca M, Venturini P. Giset. Determinants of Adenomyosis in women who underwent hysterectomy for benign gynaecological conditions:results from a prospective multi center study in Italy. . Eur J Obstet Gynaecol Reprod Biol 2009;143:103-6.

[69] Cohen I, Beyth Y, Shapiro J, Topper R, Fischman A, Cordobs $\mathrm{M}$, et al. High frequency of Adenomyosis in postmenopausal breast cancer patients treated with tamoxifen. Gynaecol Obstet Investig 1997;44:200-5.

[70] Bergeron C, Amant F, Ferenczy A. Pathology and physio pathology of Adenomyosis. Best Pract Res Clin Obstet Gynaecol 2006;20:511-21.

[71] Varras M, Polyzos D, Akrivis C. Effects of tamoxifen on the human female genital tract :review of the literature. Eur J Gynaecol Obstet 2003;24:25868.

[72] Huang PC,LiWF,Liao PC,Sun CW, Tsai EM, WangSL. Risk for estrogen dependent diseases in relation to phthalate exposure and polymorphisms of CYP17A1 and estrogen receptor gene. Environ Sci Pollut Res Int 2014;23:13964-73.

[73] Huang PC, Tsai EM, Li WF, Liao PC, Chung MC, Wang $\mathrm{YH}$, et al. Association between phthalate exposure and and glutathione S transferase M1 polymorphisms in Adenomyosis, leiomyoma and endometriosis. Hum Reprod 2010;35:386-94.

[74] Yen CF, Huang SJ, Lee CL, Wang HS, Liao SK. Molecular characterization of the endometrium in uterine Adenomyosis and its biochemical microenvirinment. Reprod Sci 2017;24:134661.

[75] Cedar H, Bergman Y. Linking DNA and histone modification:patterns and paradigm. Nat Rev Genet 2009;10:295-304.

[76] Liu X, Guo SW. Abberant immunoreactivity of deoxyribonucleic acid methyl transferase in Adenomyosis. Gynecol Obstet Invest 2012;74:100-8.

[77] Liu X, Yuan L, Kim SW. Valproic acid as a therapy for adenomyosis:a comparative case series. Repr Sci 2010;17:904-12.

[78] Liu X, Guo SW. A pilot study on the offlabel use of Valproic acid to treat adenomyosis. Fertil Steril 2008;89:246-50.

[79] Liu X, Guo SW. Valproic acid alleviates generalized hyperalgesia in mice with induced adenomyosis. J Obstet Gynaecol Res 2011;37:696-708. 
An Update on the Various Theories of the Origin of Uterine Adenomyosis -A Systematic Review

[80] Mao X, Wang Y, Carter AV, Zhen X, Guo SW. The retardation of myometrial inflammation: Reduction of uterine contractility and alleviation of general and hyperalgeasia in mice with induced Adenomyosis by levo tetrahydropalmitate g-THP and Androgro pholide . Repr Sci 2011;18:102537.

[81] Guo SW. Epigenetics of endometriosis . MolHum Reprod 2009;15:587-607.
[82] Gordts S, Koninckx P, Brosens i. Pathogenesis of deep endometriosis. Fertil Steril 2017;108:87585.

[83] Kobayashi H, Kishi Y, Matsubara S. Mechanisms underlying Adenomyosis-Related Fibrogenesis. Gynecol Obstet Invest 2020;85(1):1-12.

[84] Guo SW. The Pathogenesis of Adenomyosisvis a vis endometriosis. . JClin. Med. 2020, 9(2), 485; https://doi. org/10.3390/jcm9020485.

Citation: Dr. Kulvinder Kochar Kaur, Dr. Gautam Allahbadia, Dr. Mandeep Singh. An Update on the Various Theories of the Origin of Uterine Adenomyosis -A Systematic Review. Archives of Urology. 2020; 3(1): 31-45.

Copyright: (c) 2020 Dr. Kulvinder Kochar Kaur, Dr. Gautam Allahbadia, Dr. Mandeep Singh. This is an open access article distributed under the Creative Commons Attribution License, which permits unrestricted use, distribution, and reproduction in any medium, provided the original work is properly cited. 